\title{
GLOBAL CLASSICAL SOLUTIONS FOR REACTION-DIFFUSION SYSTEMS WITH NONLINEARITIES OF EXPONENTIAL GROWTH
}

\author{
BELGACEM REBIAI AND SAÏD BENACHOUR
}

\begin{abstract}
The aim of this study is to prove global existence of classical solutions for problems of the form $\frac{\partial u}{\partial t}-a \Delta u=-f(u, v)$, $\frac{\partial v}{\partial t}-b \Delta v=g(u, v)$ in $(0,+\infty) \times \Omega$ where $\Omega$ is an open bounded domain of class $C^{1}$ in $\mathbb{R}^{n}, a>0, b>0, a \neq b$ and $f, g$ are nonnegative continuously differentiable functions on $[0,+\infty) \times[0,+\infty)$ satisfying $f(0, \eta)=0, g(\xi, \eta) \leq C \varphi(\xi) e^{\alpha \eta^{\beta}}$ and $g(\xi, \eta) \leq \psi(\eta) f(\xi, \eta)$ for some $C>0, \alpha>0$ and $\beta \geq 1$ where $\varphi$ and $\psi$ are any nonnegative continuously differentiable functions on $[0,+\infty)$ satisfying $\varphi(0)=0$ and $\lim _{\eta \rightarrow+\infty} \eta^{\beta-1} \psi(\eta)=1$. The asymptotic behavior of the global solutions as $t$ goes to $+\infty$ is also studied. For this purpose, we use the appropriate techniques which are based on semigroups, energy estimates and Lyapunov functional methods.
\end{abstract}

\section{Introduction}

In this article, we are interested in global existence and large time behavior of classical solutions to the following reaction-diffusion system

$$
\begin{aligned}
& \frac{\partial u}{\partial t}-a \Delta u=-f(u, v) \quad \text { in } \quad(0,+\infty) \times \Omega \\
& \frac{\partial v}{\partial t}-b \Delta v=g(u, v) \quad \text { in } \quad(0,+\infty) \times \Omega .
\end{aligned}
$$

where $\Omega$ is an open bounded domain of class $C^{1}$ in $\mathbb{R}^{n}, a, b$ are positive constants and $f, g$ are nonnegative continuously differentiable functions on $[0,+\infty) \times[0,+\infty)$ satisfying the following assumptions:

$$
\begin{array}{ll}
\text { (A1) } & f(0, \eta)=g(0, \eta)=0 \quad \text { and } \quad g(\xi, 0) \geq 0 \\
\text { (A2) } & g(\xi, \eta) \leq \psi(\eta) f(\xi, \eta)
\end{array}
$$

Date: June 15, 2009.

2000 Mathematics Subject Classification. 35K45, 35K57, 35B40.

Key words and phrases. Reaction diffusion systems, Lyapunov functionals, global existence, asymptotic behavior. 
where $\psi$ is a nonnegative continuously differentiable function on $[0,+\infty)$ such that there exists a constant $\beta \geq 1$ satisfying $\lim _{\eta \rightarrow+\infty} \eta^{\beta-1} \psi(\eta)=1$,

$$
\text { (A3) } g(\xi, \eta) \leq C \varphi(\xi) e^{\alpha \eta^{\beta}}
$$

for some $C>0$ and $\alpha>0$ where $\beta$ is the same as in (A2) and $\varphi$ is any nonnegative continuously differentiable function on $[0,+\infty)$ such that $\varphi(0)=0$.

We assume that the solutions of (1.1)-(1.2) also satisfy:

- the initial conditions:

$$
u(0, x)=u_{0}(x), \quad v(0, x)=v_{0}(x) \quad \text { in } \quad \Omega,
$$

where $u_{0}, v_{0}$ are nonnegative and bounded functions.

- the homogeneous boundary conditions:

$$
\lambda_{1} u+\left(1-\lambda_{1}\right) \frac{\partial u}{\partial \nu}=0, \quad \lambda_{2} v+\left(1-\lambda_{2}\right) \frac{\partial v}{\partial \nu}=0 \text { on }(0,+\infty) \times \partial \Omega,
$$

where $\frac{\partial}{\partial \nu}$ denotes the outward normal derivative on $\partial \Omega$ and $\lambda_{1}, \lambda_{2}$ are functions of class $C^{1}$ on $\partial \Omega$ such that: $0 \leq \lambda_{i} \leq 1, i=1,2$.

Let us mention a typical example of the nonlinearities we are considering:

$$
\begin{aligned}
f(\xi, \eta) & =\xi(1+\eta) e^{\eta^{2}} \\
g(\xi, \eta) & =\xi e^{\eta^{2}} \\
\psi(\eta) & =\frac{1}{1+\eta} \\
\varphi(\xi) & =\xi .
\end{aligned}
$$

When $a=b$, under the assumptions (A1) and (A2), the existence of global classical solutions of (1.1)-(1.4) is an evident consequence of the maximum principle.

The problem on existence and uniform bounds of solutions was initially proposed by R. H. Martin when

$$
g(\xi, \eta)=f(\xi, \eta)=\xi \eta^{\beta}, \quad \beta \geq 1,
$$

with various boundary conditions and nonnegative initial data.

Firstly, N. D. Alikakos [1] obtained $L^{\infty}$-bounds of solutions of this problem subject to the homogeneous Neumann boundary conditions under the assumption $1 \leq \beta<\frac{n+2}{n}$. 
Secondly, K. Masuda [13] solved the problem when

$$
\begin{aligned}
& g(\xi, 0)=f(0, \eta)=0, \\
& g(\xi, \eta) \leq \varphi(\xi) f(\xi, \eta), \\
& g(\xi, \eta) \leq \varphi(\xi)\left(\eta+\eta^{\beta}\right), \quad \beta>0,
\end{aligned}
$$

where $\varphi$ is a monotonically increasing function on $[0,+\infty)$.

S. L. Hollis, R. H. Martin and M. Pierre [9] treated this probem and others with triangular structure for the reactive terms and proved the existence of global classical solutions under a polynomial growth assumption on $g$.

In [6] A. Haraux and A. Youkana have generalized the method of K. Masuda to handle nonlinearities $f(\xi, \eta)$ and $g(\xi, \eta)$ satisfying

$$
g(\xi, \eta)=f(\xi, \eta) \leq \xi e^{\alpha \eta^{\beta}}, \quad 0<\beta<1, \quad \alpha>0 .
$$

In [3] A. Barabanova treated the case where $\beta=1$ and obtained the global existence of classical solutions under the condition

$$
\left\|u_{0}\right\|_{\infty}<\frac{8 a b}{\alpha n(a-b)^{2}}, \quad a \neq b .
$$

In the case of $\Omega=\mathbb{R}^{n}$, M. A. Herrero, A. A. Lacey and J. J. L. Velazquez [8] proved that the Cauchy problem admits a global classical solution for any nonnegative and bounded initial data if

$$
g(\xi, \eta)=f(\xi, \eta) \leq C \varphi(\xi) e^{\alpha \eta},
$$

for some $C>0, \alpha>0$ and any continuous and nonnegative function $\varphi$ on $[0,+\infty)$ such that $\varphi(0)=0$.

Note that some investigations had the aim to weaken the assumptions on the growth of $g$ and on initial data. For instance, in [16] M. Pierre proved that, by using a structure similar to (A2), the existence of global weak solutions for reaction diffusion systems preserving positivity of the solutions with some good boundary conditions and integrable initial data. But these solutions are only integrable and one cannot apply the $L^{\infty}$-approach even if the initial data are regular.

Our paper mainly complements the investigations of [6], [3], [11] and [16]. We prove the existence of global classical solutions of (1.1)-(1.4) when the reactive terms satisfying (A1)-(A3). 
Throughout this study, we denote by $\|\cdot\|_{p}, \quad p \in[1,+\infty)$ and $\|\cdot\|_{\infty}$ the usual norms in spaces $L^{p}(\Omega)$ and $L^{\infty}(\Omega)$, respectively, defined by

$$
\|u\|_{p}=\left(\int_{\Omega}|u(x)|^{p} d x\right)^{\frac{1}{p}} \text { and }\|u\|_{\infty}=\text { ess } \sup _{x \in \Omega}|u(x)| .
$$

\section{The main results}

The aim of this study is to show that for $f$ and $g$ satisfying the assumptions (A1)-(A3), classical solutions of (1.1)-(1.4) are global for arbitrary $u_{0}$ satisfying the following restriction

$$
\left\|u_{0}\right\|_{\infty}<\frac{8 a b}{\alpha \beta n(a-b)^{2}}, \quad a \neq b .
$$

The asymptotic behavior of the solutions as the time goes to infinity is also studied.

To achieve the privious objectives, the techniques based on semigroup methods and energy estimates are used. The idea for solving the global existence of solutions for the problem (1.1)-(1.4) is a Lyapunov functional. Similar functionals appeared in [6], [3] and [11] .

Theorem 2.1. Under the assumptions (A1)-(A3), the solutions of (1.1)-(1.4) are global and uniformly bounded on $[0,+\infty) \times \Omega$.

The study of local existence and uniqueness of solution $(u, v)$ of the problem (1.1)-(1.4) follows from the basic existence theory for abstract parabolic semilinear equations (see, e.g., [2], [7], [9] and [15]). As a consequence there exists a $T^{*} \in(0,+\infty]$ such that (1.1)-(1.4) has a unique classical solution on $\left[0, T^{*}\right) \times \Omega$. Furthermore, if $T^{*}<+\infty$, then

$$
\lim _{t \uparrow T^{*}}\left(\|u(t)\|_{\infty}+\|v(t)\|_{\infty}\right)=+\infty .
$$

Therefore, if there exists a positive constant $C$ such that

$$
\|u\|_{\infty}+\|v\|_{\infty} \leq C
$$

for all $t$ in $\left[0, T^{*}\right)$, then $T^{*}=+\infty$.

Since the initial conditions are nonnegative, then from (A1) and (A2), we conclude that the nonnegativity of the solutions and the total mass of the components are preserved with time.

Since $f \geq 0, u$ satisfies the maximum principle, i.e.,

$$
\|u(t)\|_{\infty} \leq\left\|u_{0}\right\|_{\infty} \text { for all } t \in\left[0, T^{*}\right) .
$$

Based on that, the problem of global existence reduces to establish the uniform boundedness of $v$ in $\left[0, T^{*}\right)$. By $L^{p}$-regularity theory for parabolic operator (see, e.g., [12]) it follows that it is sufficient to derive a 
uniform estimate of $\|g(u, v)\|_{p}$ on $\left[0, T^{*}\right)$ for some $p>\frac{n}{2}$.

The proof of Theorem 2.1 is based on the following key proposition.

Proposition 2.2. Suppose that the assumptions (A1)-(A3) are fulfilled. For every classical solution $(u, v)$ of $(1.1)-(1.4)$ on $\left[0, T^{*}\right) \times \Omega$, let the function

$$
L: t \longmapsto \int_{\Omega}\left[\delta u+(M-u)^{-\gamma} e^{\alpha p(v+1)^{\beta}}\right](t, x) d x,
$$

where $\alpha, \beta, \gamma, \delta, p$ and $M$ are positive constants such that

$$
\beta \geq 1, \quad\left\|u_{0}\right\|_{\infty}<M<\frac{2 \gamma}{\alpha \beta n} \text { and } \gamma=\frac{4 a b}{(a-b)^{2}} .
$$

Then, there exists $\delta>0$ and $p>\frac{n}{2}$ such that

$$
L \text { is nonincreasing on }\left[0, T^{*}\right) \text {. }
$$

\section{Proofs of the main results}

Before proving the Proposition 2.2 we first need the following lemma.

Lemma 3.1. Let $(u, v)$ be a solution of (1.1)-(1.4) on $\left[0, T^{*}\right) \times \Omega$, then under the assumptions (A1)-(A3), we have

$$
\int_{\Omega} f(u(x, t), v(x, t)) d x \leq-\frac{d}{d t} \int_{\Omega} u(x, t) d x
$$

and there exists $\delta_{1}>0$ and $p>\frac{n}{2}$ such that

$$
\int_{\Omega}\left[\alpha p \beta M(v+1)^{\beta-1} g(u, v)-\gamma f(u, v)\right] e^{\alpha p(v+1)^{\beta}} d x \leq \delta_{1} \int_{\Omega} f(u, v) d x,
$$

where $\alpha, \beta, \gamma$ and $M$ are positive constants satisfying (2.2).

Proof of Lemma 3.1. It suffices to integrate the both sides of (1.1) satisfied by $u$ on $\Omega$, to obtain (3.1).

Now, from the conditions $(2.2)$ we get $\frac{n}{2}<\frac{\gamma}{\alpha \beta M}$, so we can choose $p$ such that $\frac{n}{2}<p<\frac{\gamma}{\alpha \beta M}$.

According to the assumption (A2), we have

$$
\begin{aligned}
& {\left[\alpha p \beta M(v+1)^{\beta-1} g(u, v)-\gamma f(u, v)\right] e^{\alpha p(v+1)^{\beta}} } \\
\leq & {\left[\alpha p \beta M(v+1)^{\beta-1} \psi(v)-\gamma\right] e^{\alpha p(v+1)^{\beta}} f(u, v) . }
\end{aligned}
$$

Since $\alpha p \beta M<\gamma$ and $(\eta+1)^{\beta-1} \psi(\eta)$ goes to 1 as $\eta \rightarrow+\infty$, there exists $\eta_{0}>0$ such that for all $\eta>\eta_{0}$, we obtain

$$
\left[\alpha p \beta M(\eta+1)^{\beta-1} \psi(\eta)-\gamma\right] e^{\alpha p(\eta+1)^{\beta}} f(\xi, \eta) \leq 0 .
$$


On the other hand, if $\eta$ is in the compact interval $\left[0, \eta_{0}\right]$, then the continuous function

$$
\eta \longmapsto\left[\alpha p \beta M(\eta+1)^{\beta-1} \psi(\eta)-\gamma\right] e^{\alpha p(\eta+1)^{\beta}}
$$

is bounded. So that (3.2) immediately follows.

We proceed now to the proof of Proposition 2.2.

Proof of Proposition 2.2. Differentiating $L(t)$ with respect to $t$ and using the Green formula, one obtains

$$
\frac{d}{d t} L(t)=\delta \frac{d}{d t} \int_{\Omega} u(x, t) d x+I+J
$$

where

$$
\begin{aligned}
& I=\int_{\partial \Omega}\left[a \gamma \frac{\partial u}{\partial \nu}+b \alpha p \beta(M-u)(v+1)^{\beta-1} \frac{\partial v}{\partial \nu}\right](M-u)^{-\gamma-1} e^{\alpha p(v+1)^{\beta}} d s \\
& -\int_{\Omega}\left[a \gamma(1+\gamma)|\nabla u|^{2}+\alpha p \beta \gamma(a+b)(M-u)(v+1)^{\beta-1} \nabla u \nabla v\right. \\
& \left.+\quad \operatorname{b\alpha p} \beta(M-u)^{2}\left(\beta-1+\alpha p \beta(v+1)^{\beta}\right)(v+1)^{\beta-2}|\nabla v|^{2}\right](M-u)^{-\gamma-2} e^{\alpha p(v+1)^{\beta}} d x,
\end{aligned}
$$

where $d s$ denotes the $(n-1)$-dimensional surface element and

$$
J=\int_{\Omega}\left[\alpha p \beta(M-u)(v+1)^{\beta-1} g(u, v)-\gamma f(u, v)\right](M-u)^{-\gamma-1} e^{\alpha p(v+1)^{\beta}} d x .
$$

We now take advantage of (1.4) and $\beta \geq 1$, to obtain that

$$
I \leq-\int_{\Omega} Q(\nabla u, \nabla v)(M-u)^{-\gamma-2} e^{\alpha p(v+1)^{\beta}} d x
$$

where

$$
\begin{aligned}
Q(\nabla u, \nabla v)= & a \gamma(1+\gamma)|\nabla u|^{2}+\alpha p \beta \gamma(a+b)(M-u)(v+1)^{\beta-1} \nabla u \nabla v \\
& +b\left(\alpha p \beta(M-u)(v+1)^{\beta-1}\right)^{2}|\nabla v|^{2}
\end{aligned}
$$

is a quadratic form with respect to $\nabla u$ and $\nabla v$.

The discriminant of $Q$ is given by

$$
D=\gamma\left(\alpha p \beta(M-u)(v+1)^{\beta-1}\right)^{2}\left[\gamma(a-b)^{2}-4 a b\right] .
$$

From conditions $(2.2)$, we have $Q(\nabla u, \nabla v) \geq 0$ and consequently

$$
I \leq 0 \text {. }
$$

Concerning the term $J$, since $0 \leq u \leq\left\|u_{0}\right\|_{\infty}<M$, we observe that $J \leq\left(M-\left\|u_{0}\right\|_{\infty}\right)^{-\gamma-1} \int_{\Omega}\left[\alpha p \beta M(v+1)^{\beta-1} g(u, v)-\gamma f(u, v)\right] e^{\alpha p(v+1)^{\beta}} d x$. 
Thanks to (3.2), we get $\delta_{1}>0$ such that

$$
J \leq \delta_{1}\left(M-\left\|u_{0}\right\|_{\infty}\right)^{-\gamma-1} \int_{\Omega} f(u, v) d x .
$$

Let $\delta=\delta_{1}\left(M-\left\|u_{0}\right\|_{\infty}\right)^{-\gamma-1}$ and using (3.1), we obtain

$$
J \leq-\delta \frac{d}{d t} \int_{\Omega} u(x, t) d x
$$

From (3.3)-(3.5), we conclude that

$$
\frac{d}{d t} L(t) \leq 0
$$

This concludes the proof of Proposition 2.2.

We can now proof the Theorem 2.1.

Proof of Theorem 2.1. Let $p$ be the same as in Proposition 2.2. Since $M^{-\gamma} \leq(M-\xi)^{-\gamma}$ for all $\xi \in\left[0,\left\|u_{0}\right\|_{\infty}\right]$, it follows that

$$
\|g(u, v)\|_{p}^{p}=\int_{\Omega}|g(u, v)|^{p} d x \leq M^{\gamma} K^{p} L(t)
$$

where

$$
K=\max _{0 \leq \xi \leq\left\|u_{0}\right\|_{\infty}} \varphi(\xi) .
$$

By Proposition 2.2, we deduce

$$
\begin{aligned}
\|g(u, v)\|_{p}^{p} & \leq M^{\gamma} K^{p} L(0) \\
& \leq|\Omega| M^{\gamma} K^{p}\left[\delta\left\|u_{0}\right\|_{\infty}+\left(M-\left\|u_{0}\right\|_{\infty}\right)^{-\gamma} e^{\alpha p\left\|v_{0}+1\right\|_{\infty}^{\beta}}\right] .
\end{aligned}
$$

Hence $g(u(t,),. v(t,)$.$) is uniformly bounded in L^{p}(\Omega)$ for all $t \in\left[0, T^{*}\right)$ with $p>\frac{n}{2}$. Using the regularity results for solutions of parabolic equations in [12], we conclude that the solutions of (1.1)-(1.4) are uniformly bounded on $[0,+\infty) \times \Omega$.

\section{Asymptotic behavior of the solutions}

In this section, we are dealing with the large time behavior of nonnegative and global classical solutions of (1.1)-(1.4).

Before stating our result, let us mention some properties from the theory of semigroups. 
For each $p \in(1,+\infty)$, we can define a closed linear operator $A$ on $L^{p}(\Omega)$ with domain $D(A)$ by

$$
\begin{aligned}
A w & =-d \Delta w \text { for } w \in D(A), \\
\|w\|_{2, p} & \leq C\left(\|w\|_{p}+\|A w\|_{p}\right), \quad \text { for } \quad w \in D(A), \quad \text { and } \\
D(A) & =\left\{w \in W^{2, p}(\Omega): \lambda w+(1-\lambda) \frac{\partial w}{\partial \nu}=0 \quad \text { on } \quad \partial \Omega\right\},
\end{aligned}
$$

where $\|\cdot\|_{2, p}$ is the norm of the usual Sobolev space $W^{2, p}(\Omega), d, C$ are positive constants and $\lambda$ is a function of class $C^{1}$ on $\partial \Omega$ such that $0 \leq \lambda \leq 1$.

It is well known that $-A$ generates an analytic semigroup of bounded linear operators $\left\{e^{-t A}\right\}_{t \geq 0}$ on $L^{p}(\Omega)$ and that

$$
\left\|A^{m} e^{-t A} w\right\|_{p} \leq C_{m} t^{-m}\|w\|_{p}, \quad \text { for } \quad t>0, \quad w \in L^{p}(\Omega),
$$

with some positive constant $C_{m}$ for $m=0,1,2, \ldots$.

Using the interpolation theorem (see, e.g., [14]), we have

$$
\left\|\nabla\left(e^{-t A}-e^{-s A}\right) w\right\|_{p}^{2} \leq C\left\|\left(e^{-t A}-e^{-s A}\right) w\right\|_{p}\left\|A\left(e^{-t A}-e^{-s A}\right) w\right\|_{p},
$$

for $0<s \leq t, w \in L^{p}(\Omega)$ and some positive constant $C$.

Lemma 4.1. For a function $h$ in $L^{q}\left((0,+\infty), L^{p}(\Omega)\right)$ with $q>2$, we define the function $w$ by:

$$
w(t)=e^{-t A} w_{0}+\int_{0}^{t} e^{-(t-\sigma) A} h(\sigma) d \sigma
$$

where $w_{0}$ is a nonnegative function in $L^{\infty}(\Omega)$.

Then, for $0<s \leq t$, we have

$$
\|\nabla w(t)-\nabla w(s)\|_{p} \leq C(t s)^{\frac{-1}{2}}(t-s)^{\frac{1}{2}}+C(t-s)^{\theta}\left(\int_{0}^{t}\|h(\sigma)\|_{p}^{q} d \sigma\right)^{\frac{1}{q}}
$$

where $\theta=\frac{1}{2}-\frac{1}{q}$.

Proof of Lemma 4.1. From (4.3), we have

$$
\begin{aligned}
\nabla w(t)-\nabla w(s)= & \nabla\left(e^{-t A}-e^{-s A}\right) w_{0} \\
& +\int_{0}^{s} \nabla\left(e^{-(t-\sigma) A}-e^{-(s-\sigma) A}\right) h(\sigma) d \sigma \\
& +\int_{s}^{t} \nabla e^{-(t-\sigma) A} h(\sigma) d \sigma \\
= & I_{1}+I_{2}+I_{3} .
\end{aligned}
$$


To estimate $I_{1}$, we use (4.2) and (4.1) with $m=0,2$,

$$
\begin{aligned}
\left\|I_{1}\right\|_{p} & =\left\|\nabla\left(e^{-t A}-e^{-s A}\right) w_{0}\right\|_{p} \\
& \leq C\left\|w_{0}\right\|_{p}^{\frac{1}{2}}\left\|A\left(e^{-t A}-e^{-s A}\right) w_{0}\right\|_{p}^{\frac{1}{2}} \\
& \leq C\left\|w_{0}\right\|_{p}^{\frac{1}{2}}\left(\int_{s}^{t}\left\|A^{2} e^{-\sigma A} w_{0}\right\|_{p} d \sigma\right)^{\frac{1}{2}} \\
& \leq C(t s)^{\frac{-1}{2}}(t-s)^{\frac{1}{2}}\left\|w_{0}\right\|_{p} .
\end{aligned}
$$

The term $I_{2}$ is estimated by using (4.2), (4.1) with $m=0,2$ and the Hölder inequality,

$$
\begin{aligned}
\left\|I_{2}\right\|_{p} & \leq \int_{0}^{s}\left\|\nabla\left(e^{-(t-\sigma) A}-e^{-(s-\sigma) A}\right) h(\sigma)\right\|_{p} d \sigma \\
& \leq C \int_{0}^{s}\|h(\sigma)\|_{p}^{\frac{1}{2}}\left\|A\left(e^{-(t-\sigma) A}-e^{-(s-\sigma) A}\right) h(\sigma)\right\|_{p}^{\frac{1}{2}} d \sigma \\
& \leq C \int_{0}^{s}\|h(\sigma)\|_{p}^{\frac{1}{2}}\left(\int_{s-\sigma}^{t-\sigma}\left\|A^{2} e^{-\xi A} h(\sigma)\right\|_{p} d \xi\right)^{\frac{1}{2}} d \sigma \\
& \leq C(t-s)^{\frac{1}{2}} \int_{0}^{s}(t-\sigma)^{\frac{-1}{2}}(s-\sigma)^{\frac{-1}{2}}\|h(\sigma)\|_{p} d \sigma \\
& \leq C(t-s)^{\frac{1}{2}}\left(\int_{0}^{s}(t-\sigma)^{\frac{-q}{2(q-1)}}(s-\sigma)^{\frac{-q}{2(q-1)}} d \sigma\right)^{\frac{q-1}{q}}\left(\int_{0}^{s}\|h(\sigma)\|_{p}^{q} d \sigma\right)^{\frac{1}{q}},
\end{aligned}
$$

where

$$
\begin{aligned}
& \int_{0}^{s}(t-\sigma)^{\frac{-q}{2(q-1)}}(s-\sigma)^{\frac{-q}{2(q-1)}} d \sigma=\int_{0}^{s}(t-s+\sigma)^{\frac{-q}{2(q-1)}} \sigma^{\frac{-q}{2(q-1)}} d \sigma \\
\leq & \int_{0}^{\infty}(t-s+\sigma)^{\frac{-q}{2(q-1)}} \sigma^{\frac{-q}{2(q-1)}} d \sigma \\
\leq & (t-s)^{\frac{-q}{2(q-1)}} \int_{0}^{t-s} \sigma^{\frac{-q}{2(q-1)}} d \sigma+\int_{t-s}^{\infty} \sigma^{\frac{-q}{q-1}} d \sigma \\
\leq & C(t-s)^{\frac{-1}{q-1}} .
\end{aligned}
$$

Consequently,

$$
\left\|I_{2}\right\|_{p} \leq C(t-s)^{\theta}\left(\int_{0}^{t}\|h(\sigma)\|_{p}^{q} d \sigma\right)^{\frac{1}{q}}
$$


To estimate $I_{3}$, we use (4.2), (4.1) with $m=0,1$ and the Hölder inequality,

$$
\begin{aligned}
\left\|I_{3}\right\|_{p} & \leq \int_{s}^{t}\left\|\nabla e^{-(t-\sigma) A} h(\sigma)\right\|_{p} d \sigma \\
& \leq C \int_{s}^{t}\|h(\sigma)\|_{p}^{\frac{1}{2}}\left\|A e^{-(t-\sigma) A} h(\sigma)\right\|_{p}^{\frac{1}{2}} d \sigma \\
& \leq C \int_{s}^{t}(t-\sigma)^{\frac{-1}{2}}\|h(\sigma)\|_{p} d \sigma \\
& \leq C\left(\int_{s}^{t}(t-\sigma)^{\frac{-q}{2(q-1)}} d \sigma\right)^{\frac{q-1}{q}}\left(\int_{s}^{t}\|h(\sigma)\|_{p}^{q} d \sigma\right)^{\frac{1}{q}} \\
& \leq C(t-s)^{\theta}\left(\int_{0}^{t}\|h(\sigma)\|_{p}^{q} d \sigma\right)^{\frac{1}{q}} .
\end{aligned}
$$

Collecting the above estimates, we get (4.4).

The result of this section regarding the asymptotic behavior can be stated as follows.

Theorem 4.2. Let $\Omega$ be a convex open bounded domain of class $C^{1}$ in $\mathbb{R}^{n}$. Let $(u, v)$ be any nonnegative solution of (1.1)-(1.4). Then, as $t \rightarrow+\infty$, we have

$$
\begin{aligned}
& \left\|u(t)-u^{*}\right\|_{\infty} \longrightarrow 0 \\
& \left\|v(t)-v^{*}\right\|_{\infty} \longrightarrow 0
\end{aligned}
$$

where $u^{*}$ and $v^{*}$ are nonnegative constants such that

$$
f\left(u^{*}, v^{*}\right)=g\left(u^{*}, v^{*}\right)=0
$$

Proof of Theorem 4.2. Multiplying (1.1) by $u$, integrating over $(0, t) \times$ $\Omega$ and applying the Green formula, then from (1.3) and (1.4), we get

$$
\|u(t)\|_{2}^{2}+2 a \int_{0}^{t}\|\nabla u(s)\|_{2}^{2} d s+2 \int_{0}^{t} \int_{\Omega} u(s) f(u, v)(s) d x d s \leq\left\|u_{0}\right\|_{2}^{2},
$$

from which we obtain that $\int_{0}^{t}\|\nabla u(s)\|_{2}^{2} d s$ is uniformly bounded.

In the same manner, we have

$$
\|v(t)\|_{2}^{2}+2 b \int_{0}^{t}\|\nabla v(s)\|_{2}^{2} d s \leq\left\|v_{0}\right\|_{2}^{2}+2 \int_{0}^{t} \int_{\Omega} v(s) g(u, v)(s) d x d s .
$$

On one hand, taking into account (A2), the uniform boundedness of $(u, v)$ and (3.1), we get 


$$
\begin{aligned}
\int_{0}^{t} \int_{\Omega} v(s) g(u, v)(s) d x d s & \leq \int_{0}^{t} \int_{\Omega} v(s) \psi(v(s)) f(u, v)(s) d x d s \\
& \leq C \int_{0}^{t} \int_{\Omega} f(u, v)(s) d x d s \\
& \leq C\left(\int_{\Omega} u_{0}(x) d x-\int_{\Omega} u(x) d x\right) \\
& \leq 2 C|\Omega|\left\|u_{0}\right\|_{\infty} .
\end{aligned}
$$

Consequently, $\int_{0}^{t}\|\nabla v(s)\|_{2}^{2} d s$ is uniformly bounded.

On the other hand, using the Hölder inequality, the uniform boundedness of $(u, v)$ and (3.1), we obtain

$$
\begin{aligned}
\int_{0}^{t}\|f(u, v)(s)\|_{p}^{3 p} d s & \leq \int_{0}^{t}\left(\int_{\Omega} f(u, v)(s) d x\right)\left(\int_{\Omega} f(u, v)(s)^{\frac{3 p-1}{2}} d x\right)^{2} d s \\
& \leq C \int_{0}^{t} \int_{\Omega} f(u, v)(s) d x d s \\
& \leq C\left(\int_{\Omega} u_{0}(x) d x-\int_{\Omega} u(x) d x\right) \\
& \leq 2 C|\Omega|\left\|u_{0}\right\|_{\infty},
\end{aligned}
$$

from which we obtain, by using (A2), that

$$
\begin{aligned}
\int_{0}^{t}\|g(u, v)(s)\|_{p}^{3 p} d s & \leq \int_{0}^{t}\|\psi(v(s)) f(u, v)(s)\|_{p}^{3 p} d s \\
& \leq C \int_{0}^{t}\|f(u, v)(s)\|_{p}^{3 p} d s \\
& \leq K \int_{0}^{t} \int_{\Omega} f(u, v)(s) d x d s \\
& \leq K\left(\int_{\Omega} u_{0}(x) d x-\int_{\Omega} u(x) d x\right) \\
& \leq 2 K|\Omega|\left\|u_{0}\right\|_{\infty} .
\end{aligned}
$$

Now, since $(u, v)$ is a solution of $(1.1)-(1.4)$ on $(0,+\infty) \times \Omega$, then, by using the Lemma 4.1 with $q=3 p$, we obtain that

$$
t \longmapsto\|\nabla u(t)\|_{2} \quad \text { and } \quad t \longmapsto\|\nabla v(t)\|_{2}
$$

are uniformly continuous on $[\varepsilon,+\infty)$ for every $\varepsilon>0$.

By the arguments above, we conclude that

$$
\lim _{t \rightarrow+\infty}\|\nabla u(t)\|_{2}=\lim _{t \rightarrow+\infty}\|\nabla v(t)\|_{2}=0 .
$$


From (1.1), (1.2), (1.4), (A2), the boundedness of $v$ and (3.1), we get

$$
\frac{d}{d t} \bar{u}(t) \leq 0 \quad \text { and } \quad \frac{d}{d t}\left(\left(1+\delta_{2}\right) \bar{u}(t)+\bar{v}(t)\right) \leq 0
$$

where $\bar{u}, \bar{v}$ are respectively the mean values over $\Omega$ of $u, v$ and $\delta_{2}$ is a positive constant.

Consequently, we conclude that

$$
\lim _{t \rightarrow+\infty} \bar{u}(t)=u^{*} \quad \text { and } \quad \lim _{t \rightarrow+\infty} \bar{v}(t)=v^{*},
$$

where $u^{*}$ and $v^{*}$ are nonnegative constants.

Taking into account the fact that $\{u(t)\}_{t \geq \varepsilon}$ and $\{v(t)\}_{t \geq \varepsilon}$ are relatively compact in $C(\bar{\Omega})$ (see, e.g., [5]), (4.5) and by using the PoincaréWirtinger inequality, we obtain

$$
\lim _{t \rightarrow+\infty} u(t)=u^{*} \text { and } \lim _{t \rightarrow+\infty} v(t)=v^{*}, \quad \text { in } C(\bar{\Omega}) .
$$

Since, by (3.1) and (A2), $\int_{0}^{t} \int_{\Omega} f(u, v) d x d s$ and $\int_{0}^{t} \int_{\Omega} g(u, v) d x d s$ are uniformly bounded, we have

$$
f\left(u^{*}, v^{*}\right)=g\left(u^{*}, v^{*}\right)=0 .
$$

Thus the proof of the Theorem 4.2 is completed.

Remark 4.3. If $\lim _{\eta \rightarrow+\infty} \eta^{\beta-1} \psi(\eta)=\ell$ where $\ell$ is a nonnegative constant, then we can replace the restriction (2.1) by

$$
\ell\left\|u_{0}\right\|_{\infty}<\frac{8 a b}{\alpha \beta n(a-b)^{2}}, \quad a \neq b,
$$

and we observe that if $\ell=0$, then the initial data $u_{0} \geq 0$ in $L^{\infty}(\Omega)$ is given arbitrarily.

Acknowledgments. The first author would like to thank the kind hospitality of the "Institut Elie Cartan - Nancy", where this work was done.

\section{REFERENCES}

[1] N. D. Alikakos, $L^{p}$-bounds of solutions of reaction-diffusion equations, Comm. Partial Differential Equations 4 (1979), 827-868.

[2] H. Amann, Dynamic theory of quasilinear parabolic equations - I. Abstract evolution equations, Nonlinear Anal. 12 (1988), 895-919.

[3] A. Barabanova, On the global existence of solutions of a reaction-diffusion equation with exponential nonlinearity, Proc. Amer. Math. Soc. 122 (1994), $827-831$.

[4] A. Friedman, "Partial differential equations of parabolic type", Prentice Hall Englewood Chiffs. N. J. 1964. 
[5] A. Haraux and M. Kirane, Estimations $C^{1}$ pour des problèmes paraboliques non-linéaires, Ann. Fac. Sci. Toulouse, 5 (1983), 265-280.

[6] A. Haraux and A. Youkana, On a result of K. Masuda concerning reactiondiffusion equations, Thoku Math. J. 40 (1988), 159-163.

[7] D. Henry, "Geometric theory of semilinear parabolic equations", Lecture Notes in Mathematics 840, Springer-Verlag, New York, 1981.

[8] M. A. Herrero, A. A. Lacey and J. J. L. Velazquez, Global existence for reaction-diffusion systems modelling ignition, Arch. Rational Mech. Anal. 142 (1998), 219-251.

[9] S. L. Hollis, R. H. Martin and M. Pierre, Global existence and boundedness in reaction-diffusion systems, SIAM J. Math. Anal. 18 (1987), 744-761.

[10] S. Kaplan, On the growth of solutions of quasi-linear parabolic equations, Comm. Pure Appl. Math. XVI (1963), 305-330.

[11] S. Kouachi and A. Youkana, Global existence for a class of reaction-diffusion systems, Bull. Polish Acad. Sci. Math. 49 (2001), 303-308.

[12] O. A. Ladyzenskaja, V. A. Solonnikov and N. N. Uralceva, "Linear and quasilinear equations of parabolic type", Amer. Math. Soc. 1968.

[13] K. Masuda, On the global existence and asymptotic behavior of solutions of reaction-diffusion equations, Hokkaido Math. J. 12 (1983), 360-370.

[14] S. Mizohata, "The theory of partial differential equations", Cambridge University Press 1973.

[15] A. Pazy, "Semigroups of linear operators and applications to partial differential equations", Appl. Math. Sci. 44, Springer-Verlag, New York 1983.

[16] M. Pierre, Weak solutions and supersolutions in $L^{1}$ for reaction-diffusion systems, J. Evol. Equ. 3 (2003), 153-168.

[17] J. Smoller, "Shock waves and reaction-diffusion equations", Springer-Verlag, New York 1983.

Belgacem Rebiai: Département de Mathématiques, Université de TÉBEsSA, 12002, AlgÉRIE.

Et Institut Elie Cartan, Université Henri Poincaré, F-54506 VandeuvreLÈs-Nancy Cedex, France.

E-mail address: Rebiai.Belgacem@iecn.u-nancy.fr

Saïd Benachour: Institut Elie Cartan, Université Henri Poincaré, F-54506 Vandeuvre-Lès-NanCy Cedex, France.

E-mail address: Said.Benachour@iecn.u-nancy.fr 